\title{
Variations in the Frequency of Winter Extreme Cold Days in Northern China and Possible Causalities
}

\author{
ChaOXIA YuAN AND WENMAO Li \\ Key Laboratory of Meteorological Disaster of Ministry of Education, Collaborative Innovation Center on Forecast and \\ Evaluation of Meteorological Disasters, Nanjing University of Information Science and Technology, Nanjing, China
}

(Manuscript received 11 November 2018, in final form 7 August 2019)

\begin{abstract}
Weather and climate extremes often cause devastating disasters and motivate research to improve their understanding. Here, the spatiotemporal variations in the frequency of winter extreme cold days (WECDs) in northern China (NC) were investigated. The results show that the first EOF mode reflects the spatially consistent anomalies and explains $39 \%$ of the total variance. The second EOF mode represents the east-west contrasted anomalies and explains $17 \%$ of the total variance. In the winter, when the negative Arctic Oscillation (AO) co-occurs with the stronger Siberian high $(\mathrm{SH})$, cold air from the polar region is more easily advected southward, causing the increased number of WECDs in all of NC. The co-occurrence of negative $\mathrm{AO}$ and stronger SH may be related to excessive snow in the preceding autumn over northeastern Eurasia through the local positive feedback between the snow and surface air temperatures and the troposphericstratospheric interaction. On the other hand, in the winter when the negative AO co-occurs with the weaker $\mathrm{SH}$, eastern $\mathrm{NC}$ is still under the influence of cold-air intrusion from the polar region relevant to the negative $\mathrm{AO}$, whereas western $\mathrm{NC}$ is impacted by the anomalous warm-air advection related to the weaker SH. The weakening of the SH may be caused by the El Niño teleconnection. The reproducibility of the observed variations and possible causalities of WECDs in NC by 10 coupled models participating in CMIP5 has been assessed. All the models can reproduce the observed dominant EOF modes. The relevant large-scale circulation anomalies and possible causalities can also be well simulated by the multimodel ensemble.
\end{abstract}

\section{Introduction}

Weather and climate extremes often cause catastrophes in human society and ecosystems and thus necessitate research on their underlying mechanisms. There is little doubt that increased concentrations of greenhouse gases and the related global warming play important roles in changing the intensity and frequency of extreme events (IPCC 2013). For instance, the risk of extreme hot days and the intensity and duration of drought are increasing linearly at the global scale (Easterling et al. 2000). In addition to the linear trends, extreme events also exhibit substantial temporal fluctuations (e.g., Ratnam et al. 2016; Seager et al. 2005; Dai et al. 2005). To improve the adaptation of our society to high-impact extreme events, the World Climate Research Programme (WCRP) regards them as one of the grand challenges and promotes research on their understanding, attribution, modeling, and prediction.

Corresponding author: Chaoxia Yuan, chaoxia.yuan@nuist.edu.cn
Northern China (NC) generally refers to a vast region north of $35^{\circ} \mathrm{N}$ in China. It is inhabited by approximately $35 \%$ of the total population and covers approximately $60 \%$ of the total land. It often experiences winter extreme cold days (WECDs), mainly caused by cold-air intrusion from high latitudes and the polar region (Tao 1957; Ding 1990). Frequent occurrences of WECDs often lead to tremendous damage to local agriculture, infrastructure, and economic activities. The frequent WECDs in NC in 2010 affected 11.6 million people, damaged 1.25 million ha of cropland and caused direct economic loss of 1.4 billion U.S. dollars (Song et al. 2010). Hence, it is crucial to ascertain the causalities of WECDs for better prediction and mediation.

As it is located in East Asia, the winter climate in NC is predominantly influenced by the East Asian winter monsoon (EAWM; Boyle and Chen 1987; Ding 1990; Chen and Kang 2006; Wei et al. 2011; Takaya and Nakamura 2013; Li et al. 2014). Near the surface, the EAWM is manifested as the southward airflow between the cold Siberian high $(\mathrm{SH})$ in the Eurasian continent and 
the warm Aleutian low over the North Pacific (Ding and Krishnamurti 1987; Ding et al. 1994). The intensity of the $\mathrm{SH}$ is closely related to the strength of the EAWM and is thus often adopted to represent the EAWM variations (e.g., Guo 1994; Zhang et al. 1997; Gong et al. 2002; Jhun and Lee 2004). When the SH is stronger, the northwesterlies along its northeastern edge become stronger and advect more cold air from the higher latitudes to East Asia, causing a colder winter climate. Similarly, a deeper Aleutian low can strengthen the EAWM by enhancing the zonal pressure gradient with the $\mathrm{SH}$ and thus the southward airflow (e.g., Jhun and Lee 2004). Above the surface, the EAWM is closely connected to the quasistationary trough near Japan in the middle troposphere called the East Asian trough (EAT; Wang et al. 2009; Leung and Zhou 2015; Leung et al. 2017). The prevailing sinking and radiative cooling upstream of the EAT help to build the SH in winter (Ding and Krishnamurti 1987; Ding 1990). Hence, variations in the strength and location of the EAT can cause anomalies in the SH and EAWM accordingly (e.g., Leung and Zhou 2015, 2016; Leung et al. 2017). The subtropical jet south of the EAT in the upper troposphere is another important component of the East Asian winter climate (Yang et al. 2002; Jhun and Lee 2004; Kuang et al. 2008). Strengthening of the jet often coincides with the stronger SH, the deeper Aleutian low, and the stronger EAWM (e.g., Yang et al. 2002; Kuang et al. 2008).

The Arctic Oscillation (AO; Thompson and Wallace 1998, 2000) is one of the most prominent patterns of the large-scale circulation anomalies in the Northern Hemisphere. In the winter, when the AO is in its negative phase, there are positive sea level pressure (SLP) anomalies in the polar region surrounded by negative ones in the midlatitudes. The weakened polar vortex leads to more southward intrusion of the cold polar air. The frequency of blocking over the Ural-Siberian region and that of cold surge in East Asia are significantly increased (Jeong and Ho 2005; Zhou et al. 2009; Cheung et al. 2012, 2013, 2016). As a result, the winter surface air temperatures (SATs) in eastern China and their monthly maximum and minimum in eastern $\mathrm{NC}$ are greatly reduced (Gong et al. 2001; Suo et al. 2008). Gong et al. (2001) noted that the large-scale atmospheric circulation anomalies related to the AO can impact the East Asian winter climate by modifying the strength of the SH. However, the circulation anomalies related to the AO may directly impact the EAWM, not necessarily through the SH (Wu and Wang 2002; Overland et al. 1999). In addition, the AO-EAWM relationship undergoes a strong interdecadal variation; their connection has become closer in recent decades ( $\mathrm{Li}$ et al. 2014).
In addition to the AO, there are also some other important teleconnection patterns closely related to the East Asian winter climate, such as the Eurasian pattern (Wallace and Gutzler 1981) and the Scandinavian pattern (Barnston and Livezey 1987). When the Eurasian pattern is in its positive phase, there are anomalous planetary waves in the middle troposphere meandering from western Europe to East Asia with a positive action center around Siberia and negative centers around western Europe and Japan, respectively. The SH is thus strengthened, and East Asia becomes colder than normal (e.g., Sung et al. 2009; Takaya and Nakamura 2013; Wang and Zhang 2015). The Scandinavian pattern is similar to the Eurasian pattern but is displaced farther westward, having three action centers over the eastern North Atlantic, the Scandinavia peninsula, and Siberia. The atmospheric circulation anomalies related to the positive phase of the Scandinavian pattern are conducive to anomalous cold-air accumulation around Siberia and the subsequent enhanced cold-air intrusion into East Asia (e.g., Zhang 2001; Bueh and Nakamura 2007).

The autumn snow over the Eurasian continent is other important influence on the East Asian winter climate. Both the observational analyses and numerical simulation suggest that an increase in the autumn snow over the Eurasian continent will, in turn, increase the surface albedo, reduce the absorption of solar radiation, cool the land surface, strengthen the $\mathrm{SH}$, and then decrease the SATs in East Asia in the subsequent winter (e.g., Walsh and Ross 1988; Walland and Simmonds 1996; Watanabe and Nitta 1999; Gong et al. 2002; Kumar and Yang 2003). The excessive autumn snow may also induce the negative AO through the vertical propagation of Rossby waves and the tropospheric-stratospheric interaction (e.g., Cohen and Entekhabi 1999; Gong et al. 2002; Allen and Zender 2011; Cohen et al. 2014).

El Niño-Southern Oscillation (ENSO) represents one of the largest sources of interannual variations and predictability and plays an important role in the East Asian winter climate. In the mature winter of El Niño, an anomalous anticyclone appears in the western North Pacific (Wang et al. 2000). The southwesterlies along its northwestern frame weaken the southward airflow of the EAWM, resulting in a warmer winter in eastern China (e.g., Zhang et al. 1996; Tao and Zhang 1998; Wang et al. 2000). El Niño also deepens the Aleutian low and shifts it northeastward via the Pacific-North America (PNA) teleconnection (e.g., Bjerknes 1969; McPhaden et al. 2006). This may cause the northward displacement of the EAT and thus the weaker $\mathrm{SH}$ and EAWM (Leung and Zhou 2016; Leung et al. 2017). In addition, the deeper Aleutian low can weaken the stratospheric polar vortex. Signals of the weakened 


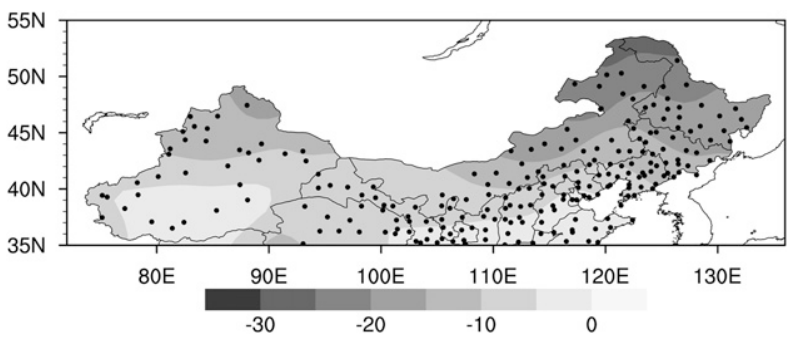

FIG. 1. Spatial distribution of the observation stations (dots) and the threshold values of WECDs (shading; ${ }^{\circ} \mathrm{C}$ ) in $\mathrm{NC}$.

vortex then propagate downward and trigger the occurrence of the negative AO (e.g., Garfinkel and Hartmann 2008; Cheung et al. 2016).

As stated above, variations in the EAWM and the winter-mean SATs have been investigated intensively. In terms of extreme cold events, there are some studies examining their linear trends and future projections under global warming (e.g., You et al. 2011; Ma et al. 2012). Additionally, some studies have focused on case studies and tried to identify the key processes contributing to specific events (e.g., Song and Wu 2017; Wen et al. 2009; Zhou et al. 2009; Wu et al. 2017). As summarized by Sillmann et al. (2017), the development of an extreme event often requires the presence of large-scale drivers. Climate models need to be able to simulate the large-scale drivers for the realistic simulation and thus reliable prediction of extreme events. However, detecting, attributing, and modeling the large-scale drivers remain a major challenge to overcome (Sillmann et al. 2017). Hence, in this study, the spatiotemporal variations in the frequency of WECDs in $\mathrm{NC}$ are investigated. The related large-scale atmospheric circulation anomalies and possible causalities are discussed to reveal the dominant large-scale drivers. Additionally, the reproducibility of the observed variations and large-scale drivers of the WECDs by the coupled models participating in phase 5 of the Coupled Model Intercomparison Project (CMIP5; Taylor et al. 2012) is assessed to estimate to what extent the models can simulate them to evaluate the potential predictability of the WECDs.

The rest of the content is organized as follows. Section 2 describes the data, the methods, and the CMIP5 models applied. Sections 3 and 4 present the spatiotemporal variations in the number of WECDs in NC. The related large-scale circulation anomalies and possible causalities are also discussed. Section 5 shows the reproducibility of the WECDs in 10 CMIP5 coupled models. Conclusions and a discussion are given in section 6 .

\section{Data and methods}

The daily temperatures from 1951 to 2014 are provided by the Chinese Meteorological Administration. A preliminary screening is conducted to exclude some stations with missing data exceeding $10 \%$ of all winter days (December-February). A total of 225 stations in $\mathrm{NC}$ are thus selected, as denoted by the dark dots in Fig. 1. The monthly NCEP-NCAR reanalysis (Kalnay et al. 1996), NOAA Global Historical Climatology Network version 2 and Climate Anomaly Monitoring System (GHCN-CAMS) Land Temperature Analysis (Fan and van den Dool 2008), and HadISST (Rayner et al. 2003) for the same period are also used. Due to the availability, the weekly NOAA snow cover extent for a shorter period of 1966-2014 is utilized (Estilow et al. 2015). Model outputs from the preindustrial experiments by 10 CMIP 5 models that provide 63 -yr necessary daily datasets are also used to examine whether the coupled models can reproduce the spatial patterns and large-scale drivers of the WECDs in NC. The models are (a) EOF1 of WECD in NC
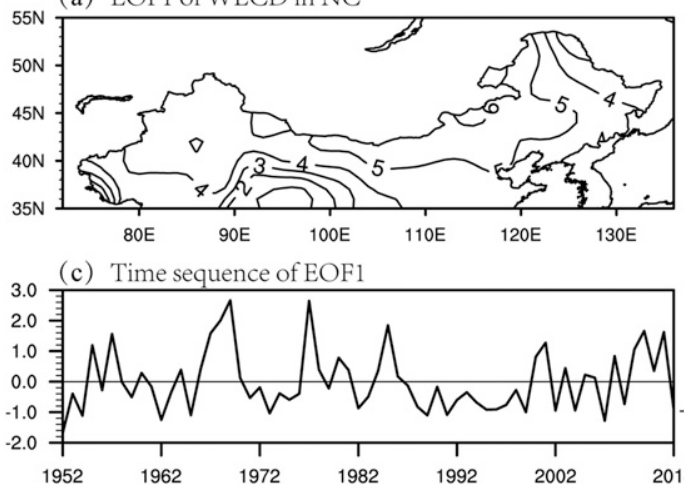

(b) EOF2 of WECD in NC

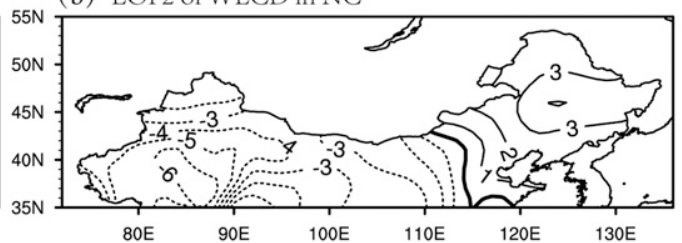

(d) Time sequence of EOF2

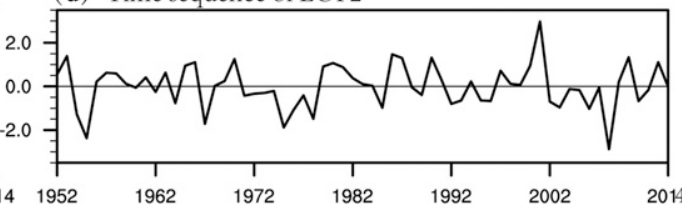

FIG. 2. (a) The first and (b) second EOF modes of the anomalies in the frequency of WECDs in NC and (c),(d) the corresponding time sequences. Each year that appears along the $x$ axis corresponds to the winter with December of the previous year and January and February of that year. 
(a) SLP, W10m and SAT Anom related to EOF1

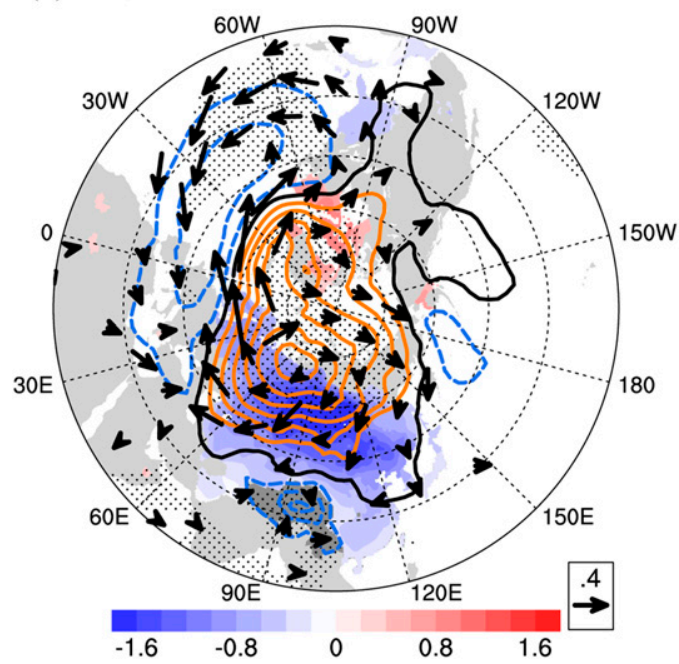

(b) SLP, W10m and SAT Anom related to EOF2

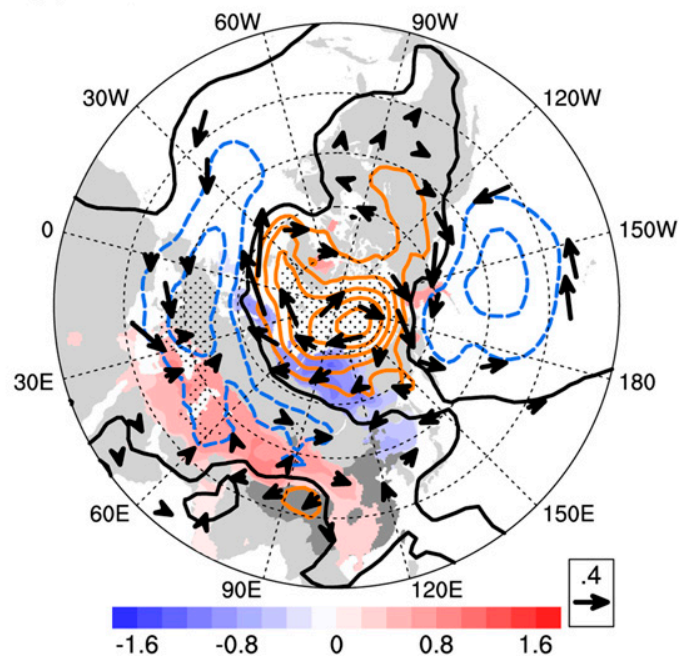

FIG. 3. December-February anomalies in the SLP (contours; interval: $0.5 \mathrm{hPa}$ ), 10 -m winds (vectors; $\mathrm{m} \mathrm{s}^{-1}$ ), and SATs (shading; ${ }^{\circ} \mathrm{C}$ ), regressed on the time sequences of (a) EOF1 and (b) EOF2. The SLP anomalies significant at the $95 \%$ confidence level are stippled, and only the $10-\mathrm{m}$ wind and SAT anomalies significant at the $95 \%$ confidence level are shown. The outermost latitude of the polar projection is $15^{\circ} \mathrm{N}$, with a meridional interval of $15^{\circ}$.

IPSL-CM5A-MR, IPSL-CM5B-LR, MIROC-ESM, MIROC-ESM-CHEM, MPI-ESM-MR, MPI-ESM-LR, INM-CM4, MRI-CGCM3, CNRM-CM5, and MPIESM-P. The preindustrial experiments in CMIP5 adopt nonevolving preindustrial conditions, including air composition, aerosol concentration, and land use. Hence, they can serve as the baseline for historical and future scenarios and be used to examine the unforced variability of the climate system in the models (Taylor et al. 2012).

Several indices are used here. The WECD at a station is defined as a day when the maximum temperature is below or equal to the 10th percentile of all the winter daily maximum temperatures at that station during the
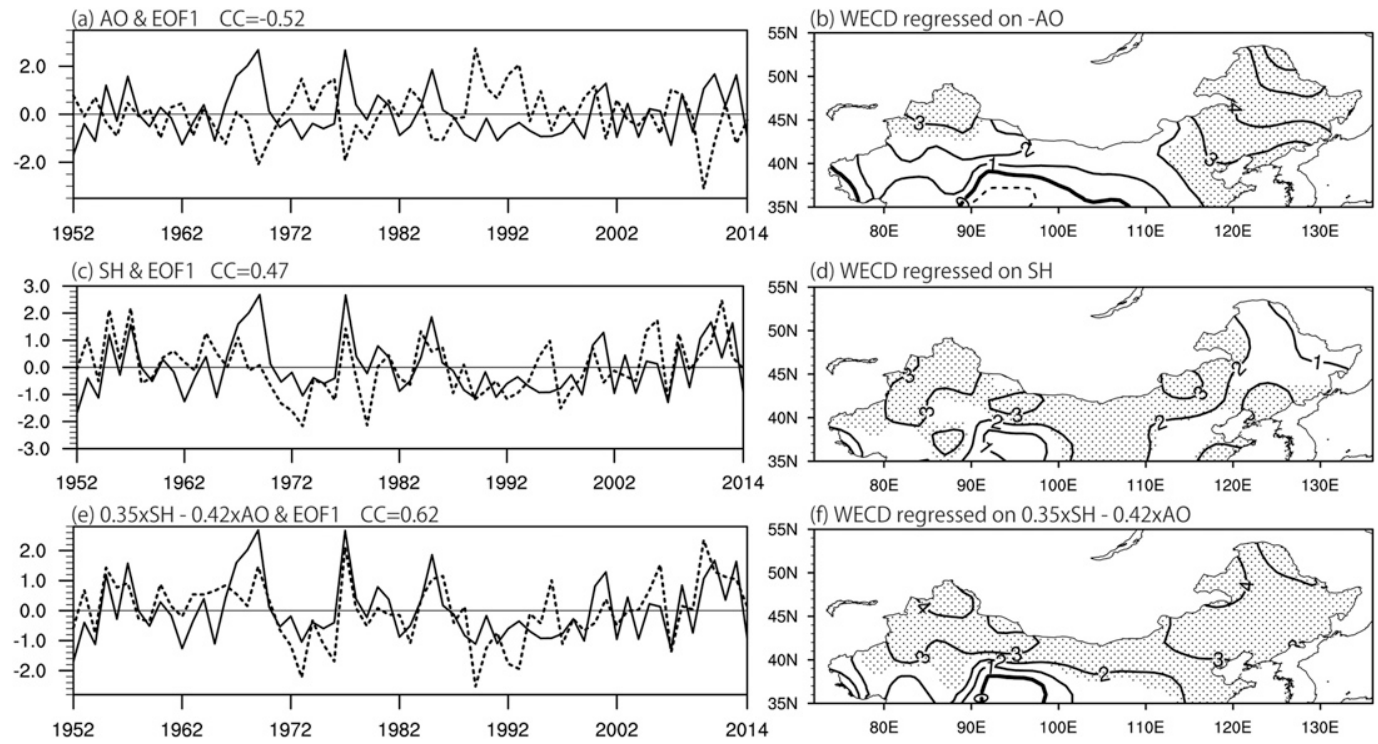

FIG. 4. December-February indices of (a) the AO (dotted), (c) the SH (dotted), and (e) their linear combination of $0.35 \mathrm{SH}-0.42 \mathrm{AO}$ (dotted), and (b),(d),(f) the corresponding regressed WECD anomalies in NC (interval: 1 day). The solid lines in (a), (c), and (e) are the time sequences of EOF1. The WECD anomalies significant at the $95 \%$ confidence level according to the two-tailed $t$ test are stippled in (b), (d), and (f). 
(b) DJF SLP, W10 and SAT Anom regressed on SON snow

(a) CC with SON Snow

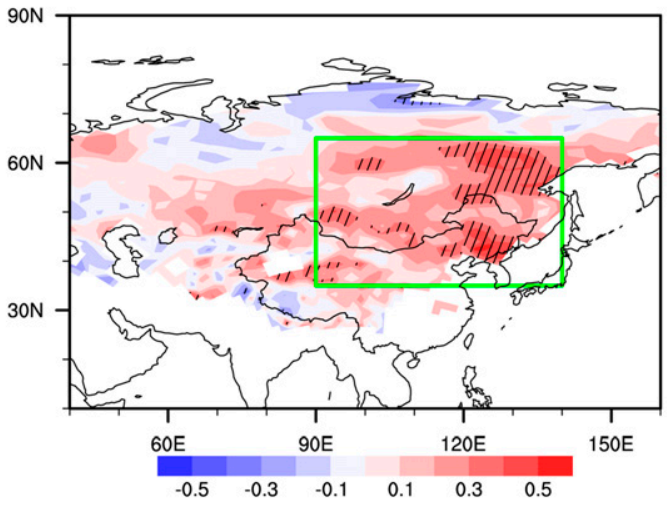

(c) SON snow vs EOF1, cc $=0.44$

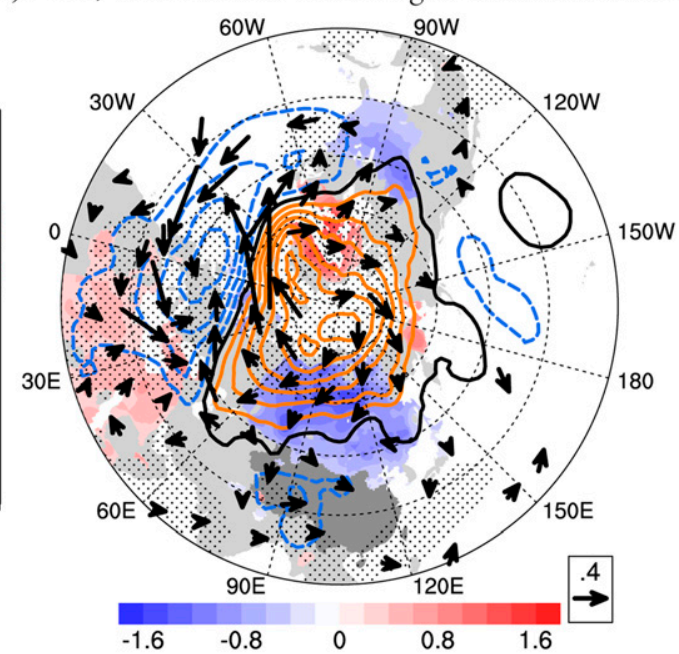

$-1.6$

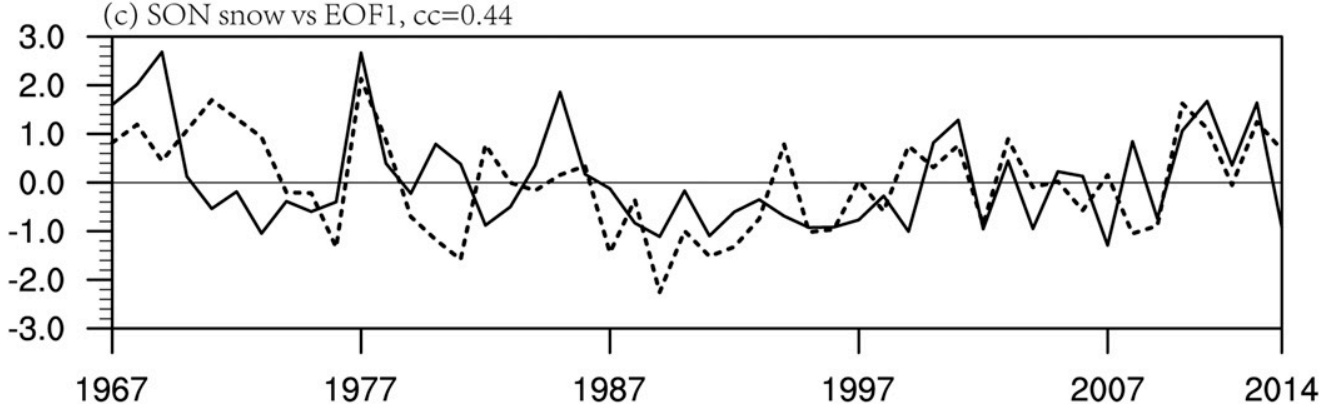

FIG. 5. (a) Correlation coefficients between September-November snow cover extent over the Eurasian continent and the time sequence of EOF1. (b) As in Fig. 3a, except that the anomalies are regressed onto (c) the September-November snow index (dotted line) represented by the snow anomalies over the green rectangular box in (a). The solid line in (c) is the time sequence of EOF1.

base period of 1961-90 (Fig. 1). The frequency of WECDs at each station in each winter can thus be calculated. The intensity of the $\mathrm{SH}$ is represented by the regional-mean SLP anomalies over $40^{\circ}-60^{\circ} \mathrm{N}, 70^{\circ}-120^{\circ} \mathrm{E}$ following Gong et al. (2002). The AO index is the time sequence of the first mode of the empirical orthogonal function (EOF) applied to the December-February SLP anomalies north of $20^{\circ} \mathrm{N}$ weighted by the grid area (e.g., Nakamura et al. 2006; Chen et al. 2014). The ENSO is represented by the Niño-3 index, which can be downloaded from the NOAA website (https:// www.esrl.noaa.gov/psd/data/correlation/nina3.data).

To focus on the year-to-year fluctuation, the longterm mean and the linear trend in all datasets are removed beforehand. The EOF analysis is applied to the anomalies of WECD frequency in NC to extract the major spatial patterns. Figure 2 shows the leading two independent modes verified by North's rule of thumb (North et al. 1982). The first mode (EOF1) reflects the spatially consistent anomalies and explains $\sim 39 \%$ of the total variances. The second mode (EOF2) represents the east-west contrasted anomalies and explains $\sim 17 \%$ of the total variances. Both modes undergo substantial temporal variations (Figs. 2c,d). To reveal the largescale circulation anomalies and possible causalities related to the major EOF modes, linear correlation and regression and composite analyses are applied. The statistical significance is tested by the two-tailed $t$ test.

\section{The large-scale circulation anomalies and possible causalities related to EOF1}

Figure 3a shows the December-February anomalies in the SLP, 10-m winds, and land SATs regressed on the time sequence of EOF1 (see Fig. 2c). It seems that when all of NC experiences more frequent WECDs, the largescale circulation anomalies in the lower troposphere are analogous to the negative phase of the AO, with the positive SLP anomalies in the polar region surrounded by the negative ones in the midlatitudes (Thompson and Wallace 1998). However, compared to the classical pattern of the negative AO, the positive SLP anomalies 
(a) CCs with SH and SAT

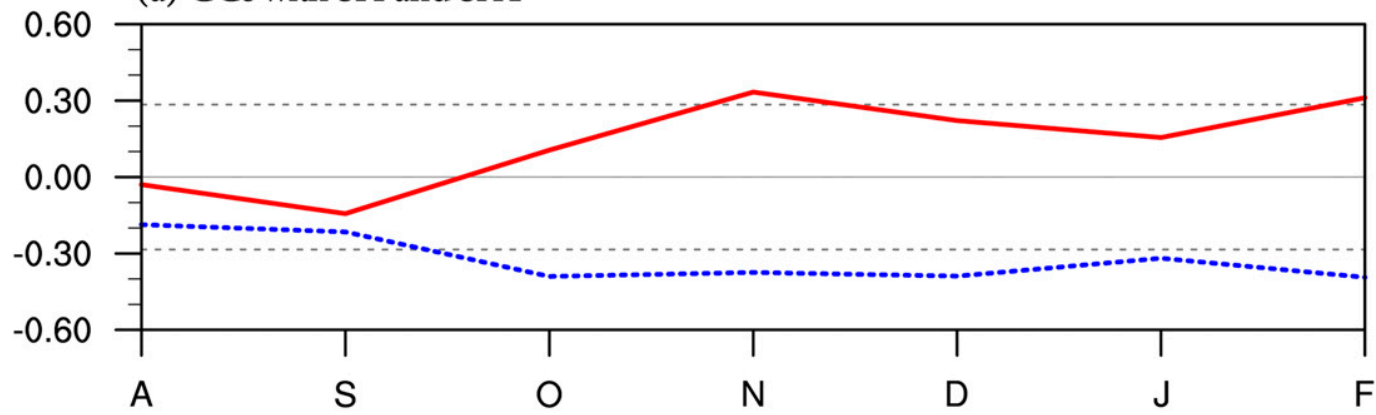

(b) CCs with vertical WAF

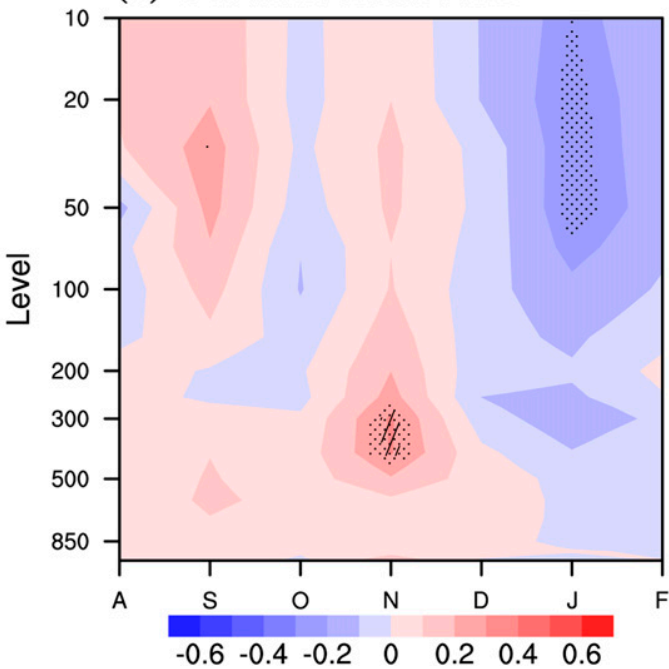

(c) CCs with GPH in polar cap

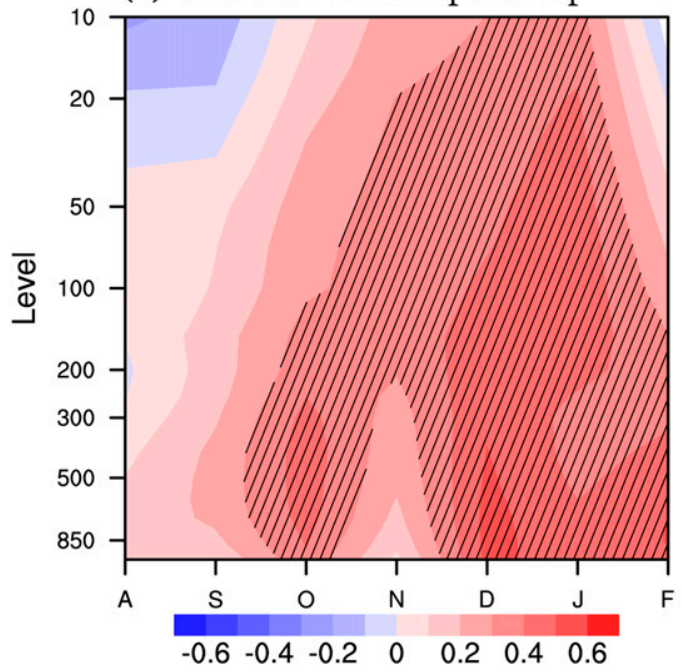

FIG. 6. Lead-lag correlation coefficients between the September-November snow index and the monthly anomalies in (a) the SLP (red solid line) and the SAT (blue dotted line) averaged over the region where the SH index is defined, (b) the vertical wave activity fluxes averaged over the circumpolar band $\left(40^{\circ}-80^{\circ} \mathrm{N}\right)$, and (c) the geopotential height averaged over the polar cap $\left(60^{\circ} \mathrm{N}\right.$ northward) from the preceding August to the subsequent February during 1967-2014. The correlation coefficients significant at the 90\% (95\%) confidence level are stippled (hatched) in (b) and (c). The gray dotted lines in (a) denote the $95 \%$ confidence level. The capital letters along the $x$ axis denote the months.

in the Eurasian sector extend too far southward and cover most of the midlatitudes. This indicates the concurrent strengthening of the SH. As discussed in previous studies (e.g., Guo 1994; Gong et al. 2001), the higher atmospheric pressures over the Arctic (Siberia) related to the negative phase of the $\mathrm{AO}$ (stronger $\mathrm{SH}$ ) can enhance the EAWM and the southward intrusion of cold air. It can be seen that a large amount of colderthan-normal air accumulates in $\mathrm{NC}$ and the region to its north (Fig. 3a), which suggests an increase in the number of WECDs in all of NC. In fact, the correlation coefficient between EOF1 and the $\mathrm{AO}$ is -0.52 and that between EOF1 and the $\mathrm{SH}$ is 0.47 , with both values significant at the $99 \%$ confidence level (Figs. 4a,c). When the negative AO occurs, the northwestern tip and eastern NC have more WECDs (Fig. 4b). In contrast, when the $\mathrm{SH}$ is stronger than normal, central, and western NC are likely to have more WECDs (Fig. 4d). This suggests that the co-occurrence of a negative AO and stronger $\mathrm{SH}$ can lead to a consistent increase in the number of WECDs in NC. If the AO and SH indices are used to predict EOF1, we can obtain the following multiple linear regression equation: $y=0.35 \mathrm{SH}-$ $0.42 \mathrm{AO}$, where both coefficients are significant at the $99 \%$ confidence level. The equation has a correlation coefficient of 0.62 with EOF1 (Fig. 4e). In addition, the WECD anomalies regressed upon $y$ show a spatially consistent increase over NC (Fig. 4f).

The large-scale circulation anomalies related to EOF1 are probably induced by the preceding autumn snow in the Eurasian continent. Figure 5a shows the correlation coefficients between EOF1 and the preceding 


\section{(a) $\mathrm{AO}$ - and $\mathrm{SH}$ -}

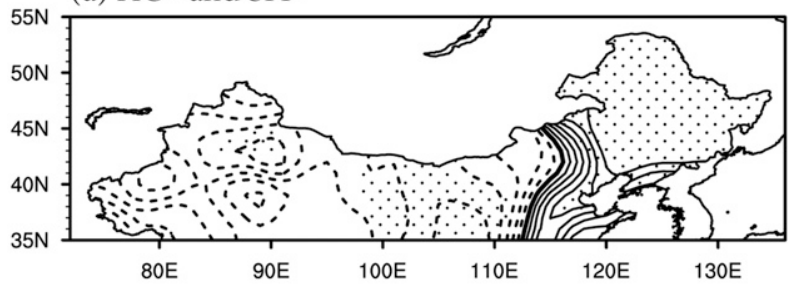

(b) AO- and El Nino

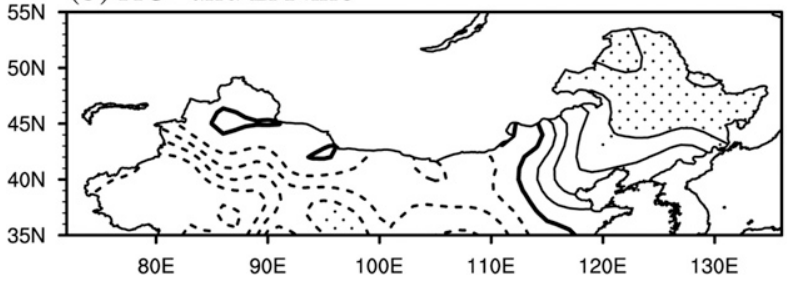

FIG. 7. Composite anomalies in the frequency of WECDs (interval: 1 day) in NC when the negative AO co-occurs with (a) the weaker SH or (b) El Niño. Anomalies significant at the $90 \%$ confidence level are stippled.

September-November snow cover extent. It is apparent that there are significant positive correlation coefficients over northeastern Eurasia. If an autumn snow index is defined as the regional-mean snow cover anomalies over $35^{\circ}-65^{\circ} \mathrm{N}, 90^{\circ}-140^{\circ} \mathrm{E}$, as denoted by the rectangular box in Fig. $5 \mathrm{a}$, it has a correlation coefficient of 0.44 with EOF1, which is significant at the $99 \%$ confidence level (Fig. 5c). Additionally, the circulation anomalies in the subsequent winter regressed on the autumn snow index are very similar to those related to EOF1 (Figs. 3a and $5 b$ ).

The delayed impacts of the autumn snow may be caused by the positive feedback between the local snow and SATs and the tropospheric-stratospheric interaction (e.g., Cohen and Entekhabi 1999; Gong et al. 2002; Cohen et al. 2007; Allen and Zender 2011). When the autumn snow cover is larger than normal, it increases the local surface albedo, reflects more incoming solar radiation, and decreases the SATs. The lower SATs help to further aggregate the snow and increase the surface albedo. The positive feedback may persist to the subsequent winter and intensify the SH by the anomalous surface cooling since the key region of the autumn snow overlaps largely with that of the $\mathrm{SH}$ (Fig. 6a). On the other hand, signals of the positive lower-tropospheric pressure anomalies may propagate upward to the stratosphere via the vertical propagation of the Rossby wave and weaken the stratospheric polar vortex (Figs. 6b,c). The negative vortex anomalies then extend downward in the subsequent winter and trigger the occurrence of the negative AO (Fig. 6c). Note that the vertical wave activity fluxes are calculated

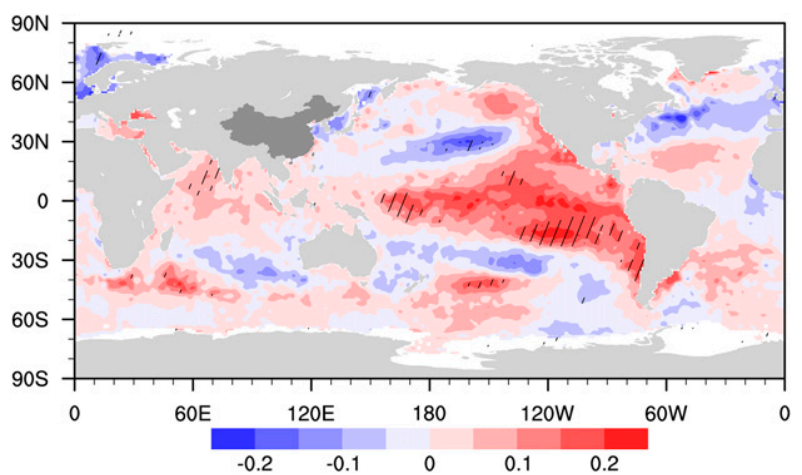

FIG. 8. December-February anomalies in the $\operatorname{SST}\left({ }^{\circ} \mathrm{C}\right)$ regressed on the time sequence of EOF2. The anomalies significant at the $95 \%$ confidence level are hatched.

following Takaya and Nakamura (2001) and are averaged over the circumpolar band of $40^{\circ}-80^{\circ} \mathrm{N}$. If the vertical wave fluxes are averaged over the key region of the autumn snow, as denoted by the green rectangular box in Fig. 5a, the upward fluxes in the preceding autumn become more statistically significant, whereas the downward fluxes in the subsequent winter become much weaker. This indicates that the upward and downward paths of the vertical Rossby wave propagation may have different geographical preferences. Nevertheless, the impacts of the autumn snow shown here are consistent with other studies where an increase in the autumn snow over the Eurasian continent is generally related to the enhanced $\mathrm{SH}$ and the negative $\mathrm{AO}$ in the subsequent winter (e.g., Cohen and Entekhabi 1999; Gong et al. 2002; Allen and Zender 2011).

\section{The large-scale circulation anomalies and possible causalities related to EOF2}

EOF2 describes the east-west contrasted anomalies in the frequency of WECDs in NC. When the frequency of WECDs increases in eastern $\mathrm{NC}$ and decreases in the west, positive SLP anomalies can be observed over the polar region, analogous to the negative phase of the AO (Fig. 3b). However, compared to the classical pattern, the negative loading over the North Atlantic shifts eastward and covers the midlatitude Eurasian continent. This reflects the concurrent weakening of the SH. As inferred from the circulation anomalies in the lower troposphere, eastern $\mathrm{NC}$ is subject to the cold-air intrusion from the polar region, while western $\mathrm{NC}$, together with a vast region of the Eurasian continent between $30^{\circ}$ and $45^{\circ} \mathrm{N}$, is under the influence of warm-air advection by the westerlies and southwesterlies along the southern frame of the negative SLP anomalies. Hence, the 

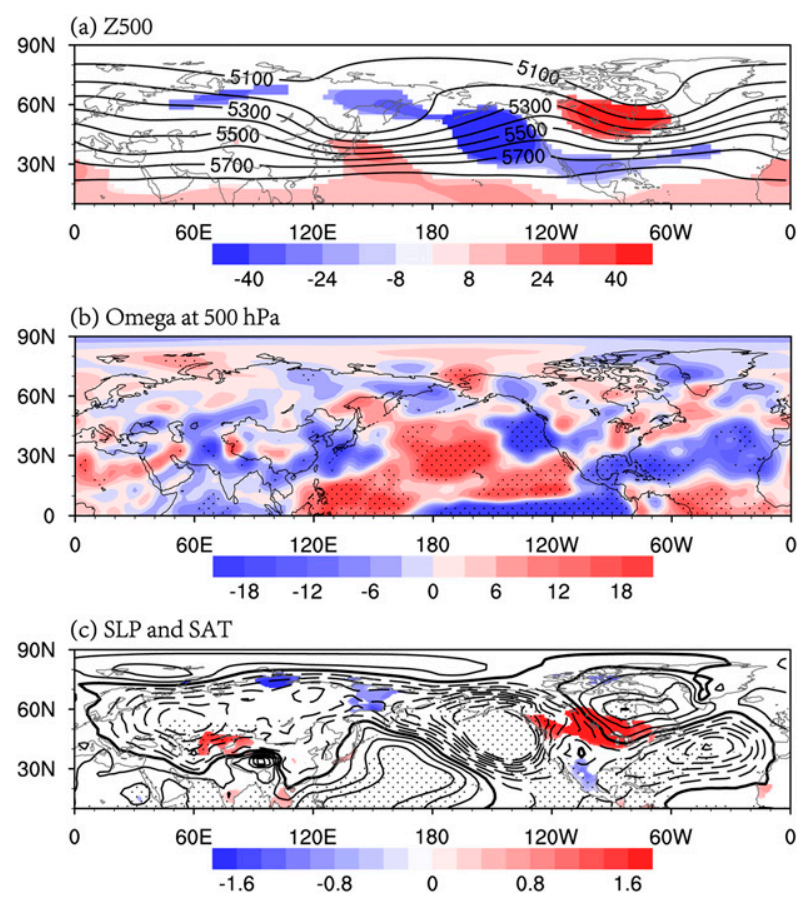

FIG. 9. December-February composited anomalies in (a) the geopotential height (shading; $\mathrm{hPa}$ ) and (b) vertical velocity (shading; $10^{-3} \mathrm{~Pa} \mathrm{~s}^{-1}$ ) at $500 \mathrm{hPa}$ and (c) the SLP (contour; interval: $0.5 \mathrm{hPa}$ ) and SAT (shading; ${ }^{\circ} \mathrm{C}$ ) during the El Niño years. The climatological geopotential heights at $500 \mathrm{hPa}$ (dark contours; gpm) are superimposed in (a). Only the anomalies significant at the $95 \%$ confidence level in the geopotential height and SAT are shown in (a) and (c), and those in the vertical velocity and SLP are stippled in (b) and (c).

frequency of WECDs tends to decrease in western NC and tends to increase in the east. To confirm the impact of the co-occurring negative $\mathrm{AO}$ and weaker $\mathrm{SH}$, we conducted composite analyses of the winters in which both the $\mathrm{AO}$ and $\mathrm{SH}$ indices were lower than -0.5 standard deviations $(1970,1979$, and 2001). The results indeed show a decrease in the number of WECDs in central and western $\mathrm{NC}$ but an increase in eastern $\mathrm{NC}$ (Fig. 7a).

There are some significant positive correlation coefficients between EOF2 and the winter SST anomalies in the eastern tropical Pacific (Fig. 8), indicating that the large-scale circulation anomalies related to EOF2 may be partly driven by the ENSO. During the winters when the Niño-3 indices were larger than one standard deviation (1958, 1966, 1973, 1983, 1987, 1992, 1998, 2010), the PNA teleconnection and the related vertical motion anomalies can be clearly seen from the North Pacific to North America (Figs. 9a-c). Leung and Zhou (2016) and Leung et al. (2017) claimed that the resultant deepening and northeastward shift of the Aleutian low can displace the EAT northward, with the positive (negative) geopotential height anomalies south (north) of Japan in the middle troposphere, as depicted in Fig. 9a. Hence, the prevailing descending motion upstream of the climatological EAT becomes weaker; the large-scale anomalous ascent can be discerned west of the climatological EAT and east of the Caspian Sea (Fig. 9b). Consequently, the SH becomes weaker (Fig. 9c) (Leung and Zhou 2015, 2016; Leung et al. 2017). Hence, the SH and ENSO have a negative correlation coefficient of -0.25 during the period of interest, which is significant at the $90 \%$ confidence level. There is also some literature discussing the impacts of the ENSO on the AO; El Niño can cause the weaker stratospheric vortex that extends downward and generates the negative AO (e.g., Li and Lau 2013; Cheung et al. 2016). However, during our study period, the ENSO showed no statistically significant
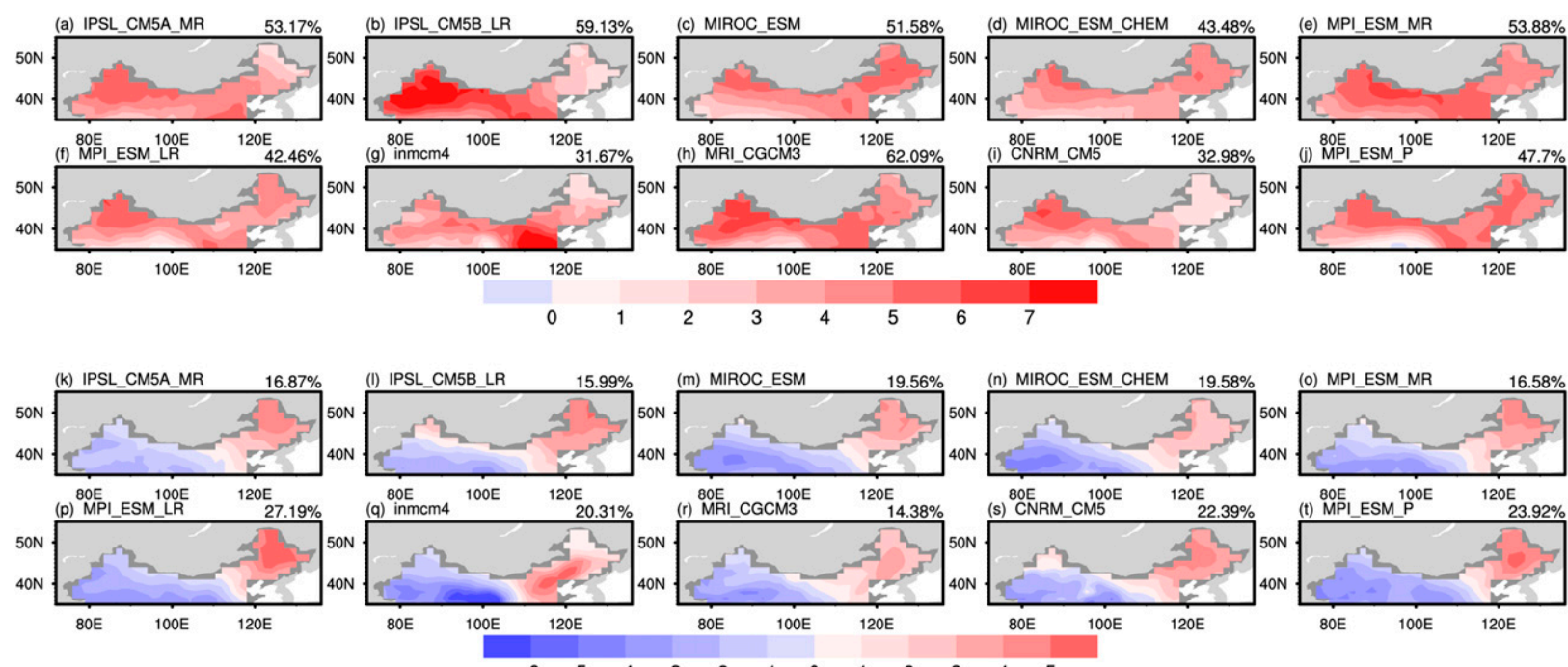

$$
\begin{array}{llllllllllll}
-6 & -5 & -4 & -3 & -2 & -1 & 0 & 1 & 2 & 3 & 4 & 5
\end{array}
$$

FIG. 10. As in Fig. 2, but for (a)-(j) the first and (k)-(t) the second EOF modes of WECDs in NC in the 10 CMIP5 coupled models. 

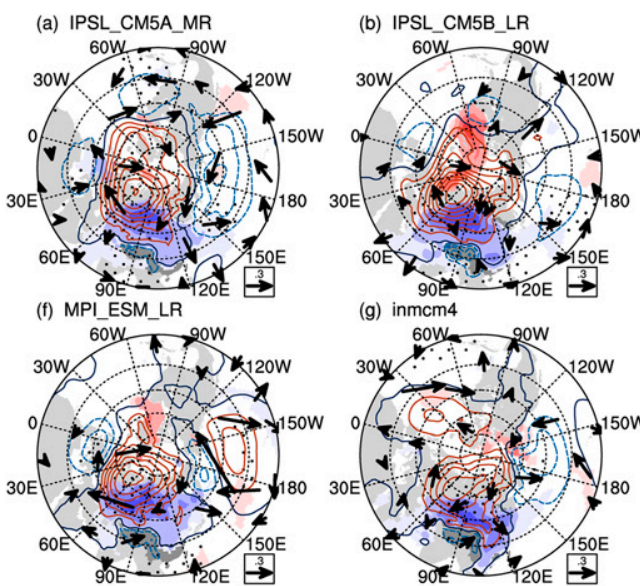

$$
\text { (g) inmcm4 }
$$
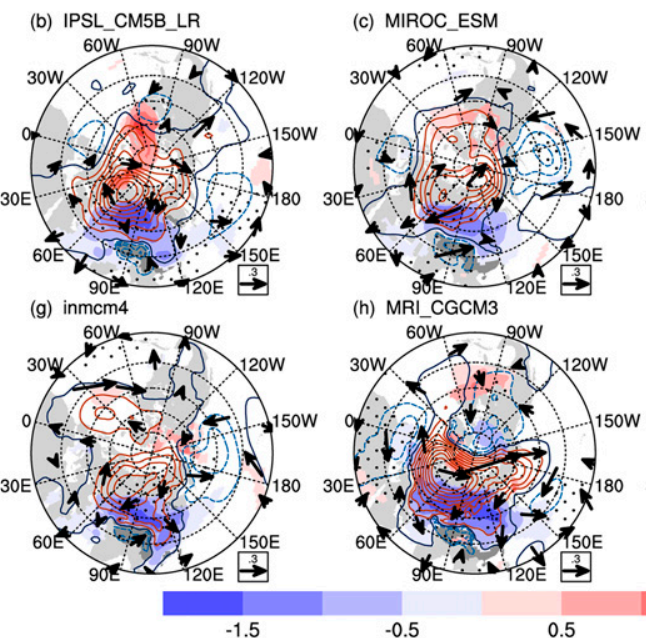

(h) MRI_CGCM3
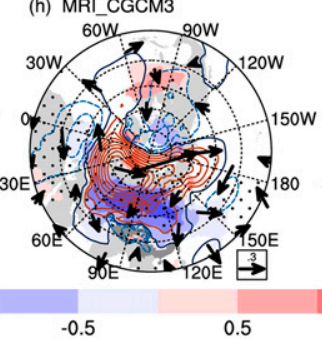

0.5

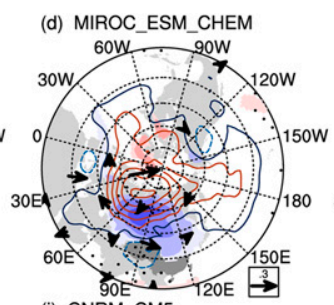

(i) CNRM_CM5
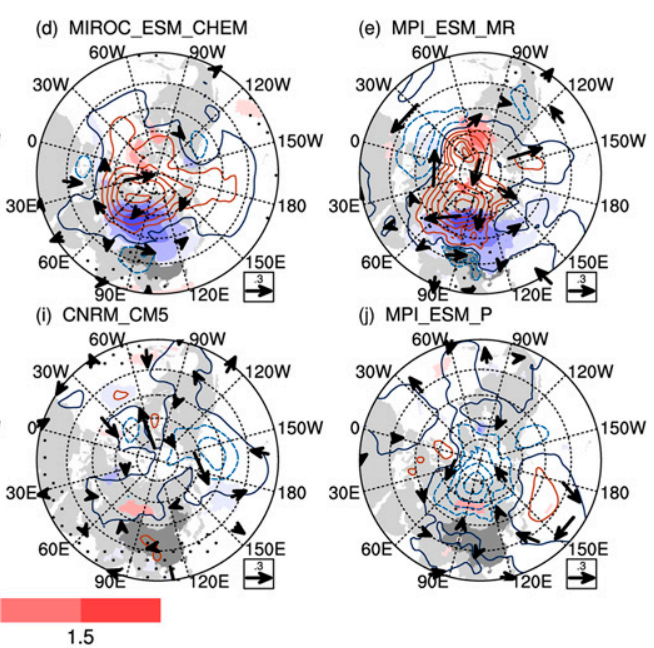
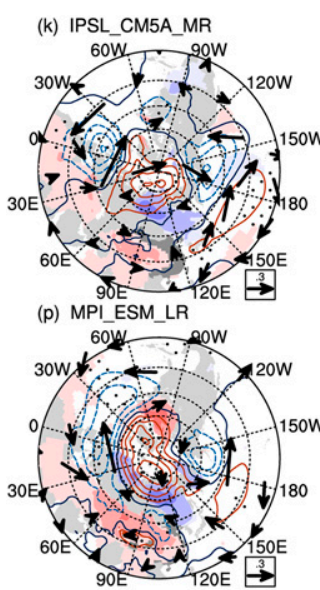

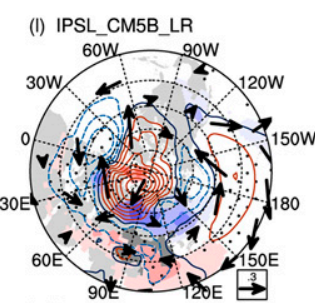

(q) inmcm 4

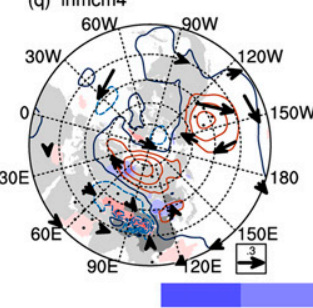

$-1.5$

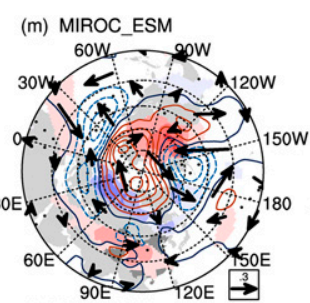

(r) MRI_CGCM3

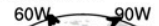

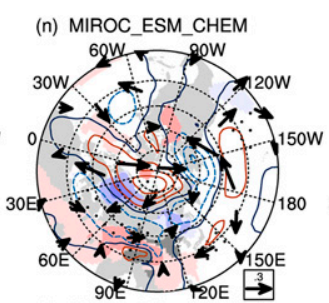

(s) CNRM CM5

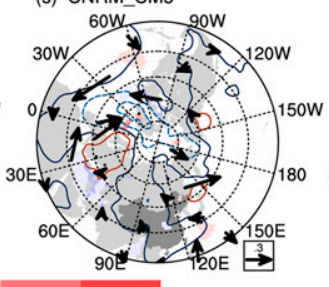

(o) MPI_ESM_MR
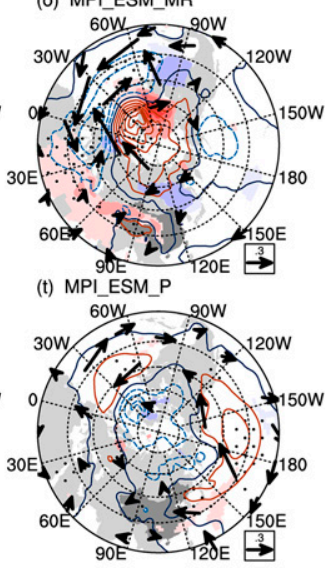

FIG. 11. As in Fig. 3, but for the anomalies regressed on the time sequences of (a)-(j) EOF1 and (k)-(t) EOF2 in the 10 CMIP5 models.

influences on the AO. The correlation coefficient between them is 0.05 , and the composite of the El Niño winters shows no significant signals of the $\mathrm{AO}$ in the lower troposphere (Fig. 9c). This is probably because the ENSO-AO relationship can be largely modified by other factors, such as the phases of the quasibiennial oscillation (e.g., Garfinkel and Hartmann 2008; Cheung et al. 2016). Nonetheless, El Niño can influence the winter climate in NC by modifying the strength of the SH. Cheung et al. (2012) showed that when El Niño co-occurred with the negative AO, the winter SATs increased in western NC but decreased in eastern NC. If we choose the winters in which the Niño-3 index was larger than one standard deviation and the AO index was negative (1958, 1966, 1987, 1998, 2010), the frequency of WECDs decreased in central and western NC but significantly increased in eastern NC (Fig. 7b), similar to the EOF2 pattern (Fig. 2b).

\section{Variations in the number of WECDs in NC in the CMIP5 coupled models}

Sections 3 and 4 analyzed the large-scale circulation anomalies and possible causalities related to the major EOF modes of WECDs in NC by using the observation and reanalysis data. Since the coupled models are now commonly adopted to simulate, predict, and even project extreme events under different representative concentration pathways of $\mathrm{CO}_{2}$ emission, it is crucial to estimate the reproducibility of the variations and large-scale drivers related to the observed extreme events. Hence, 10 coupled models in CMIP5 and their outputs from the preindustrial runs are chosen to assess their performance on the WECDs in NC. 
(a) CC between modeled and observed SLP related to EOF1

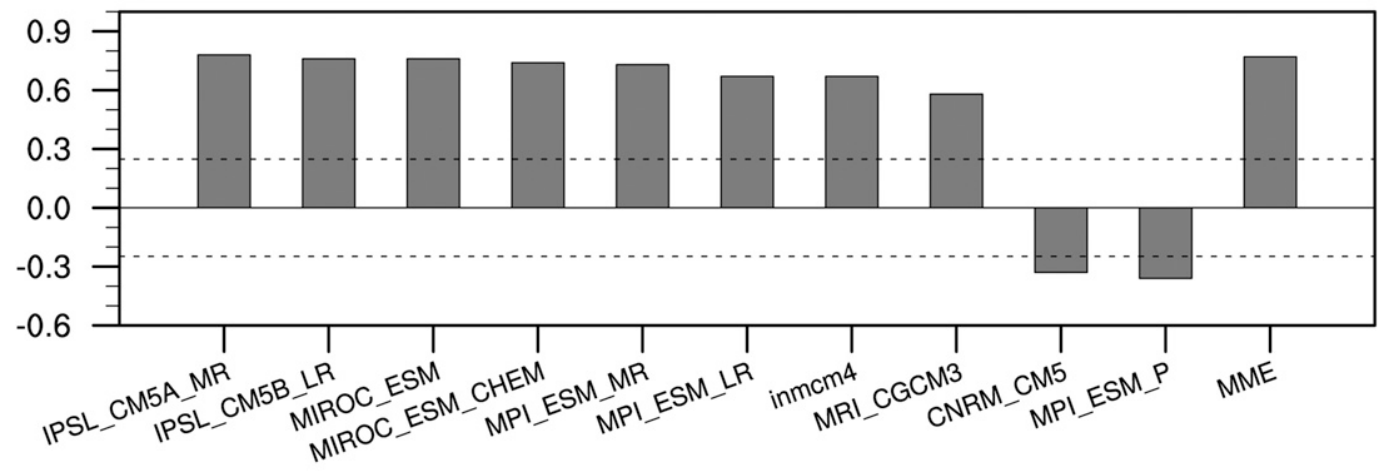

(b) CC between modeled and observed SLP related to EOF2

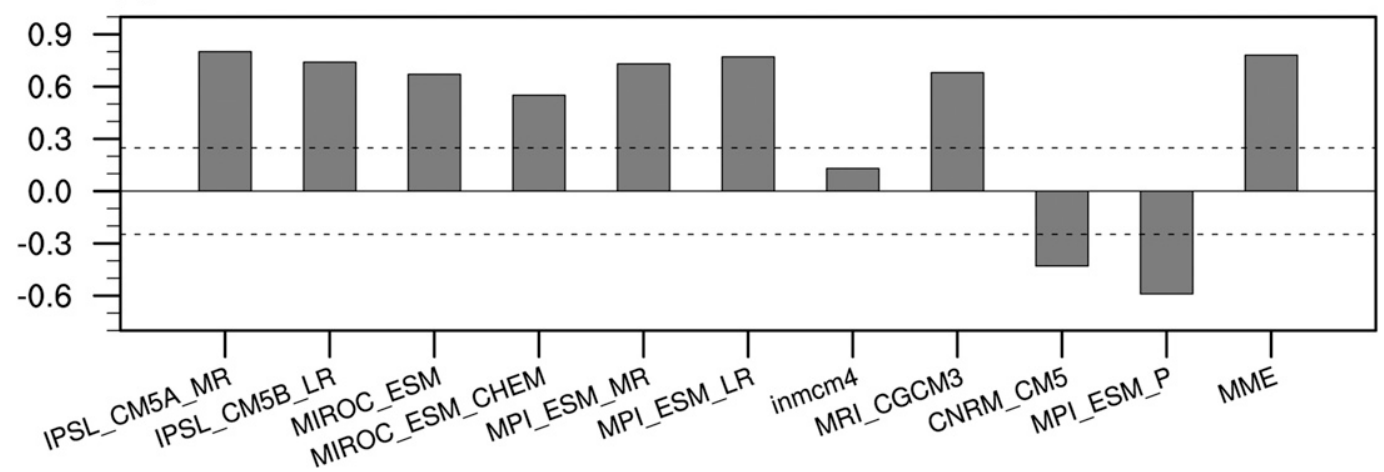

FIG. 12. The spatial correlation coefficients between the observed and modeled SLP anomalies north of $15^{\circ} \mathrm{N}$ related to (a) EOF1 and (b) EOF2 in the 10 CMIP5 models and the multimodel ensemble (MME).

The definition of a WECD and the analysis methods applied to each model output are identical to those applied to the observed and reanalysis data. The results show that the thresholds of WECDs in NC in each model are similar to the observed thresholds (figure not shown). All 10 models can successfully reproduce the observed EOF1 pattern; however, the explained variances in IPSL-CM5A-MR, IPSL-CM5B-LR, MIROC-ESM, MPI-ESM-MR, and MRI-CGCM3 are over $50 \%$, much higher than the observed 39\% (Figs. 10a-j). Most models except for CNRM-CM5 and MPI-ESM-P simulate well the co-occurrence of the negative $\mathrm{AO}$ and the stronger $\mathrm{SH}$ that leads to the spatially consistent increase in the number of WECDs in NC (Figs. 11a-j). The SLP anomalies north of $15^{\circ} \mathrm{N}$ regressed on the time sequences of EOF1 in most models have spatial correlation coefficients higher than 0.6 with respect to the observations (Fig. 12a). The spatial correlation coefficient between the multimodel ensemble and the observation is as high as 0.77 (Fig. 12a). In addition, possible impacts of the preceding autumn snow over northeastern Eurasia on the subsequent winter circulation anomalies related to EOF1 are resolved to some extent in most models and the multimodel ensemble (Fig. 13a).
The observed EOF2 pattern is also well reproduced in the 10 coupled models, with explained variances similar to those observed (Figs. 10k-t). Except for CNRM-CM5, MPI-ESM-P, and INM-CM4, most models can accurately simulate the negative phase of the $\mathrm{AO}$ and the large-scale negative SLP anomalies in the midlatitude Eurasian continent related to EOF2, analogous to the observations (Figs. 11k-t). Especially in IPSL-CM5A-MR, the modeled SLP anomalies have the highest spatial correlation coefficient $(0.80)$ with respect to the observations (Fig. 12b). The correlation coefficient between the multimodel ensemble and the observations is as high as 0.78 (Fig. 12b). In addition, most models and the multimodel ensemble simulate the possible teleconnection between EOF2 and ENSO (Fig. 13b).

\section{Conclusions and discussion}

In this study, the spatiotemporal variations in the frequency of WECDs in NC were investigated. The results show that EOF1 reflects the spatially consistent anomalies and explains $39 \%$ of the total variances. EOF2 reflects the east-west contrasted anomalies and 
(a) Modeled SON Snow related to EOF1 in CMIP5

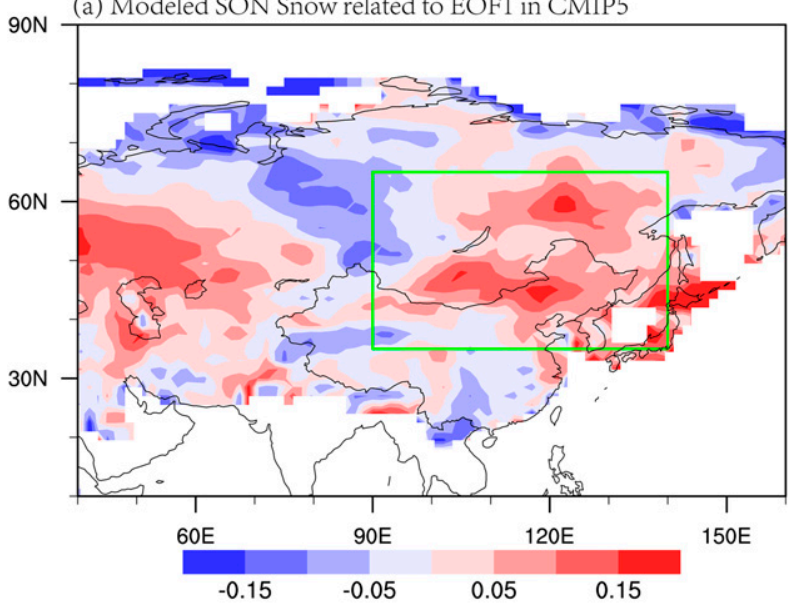

(b) Modeled DJF SST related to EOF2 in CMIP5

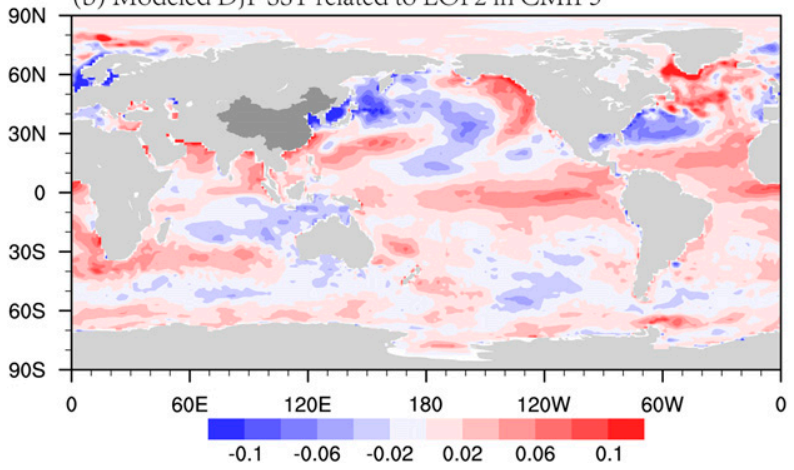

FIG. 13. The multimodel ensemble of (a) the SeptemberNovember snow-cover anomalies (ratio) related to EOF1 and (b) December-February SST anomalies $\left({ }^{\circ} \mathrm{C}\right)$ related to EOF2 in the 10 CMIP5 models.

explains $17 \%$ of the total variances. In the winter, when the $\mathrm{AO}$ is in its negative phase and the $\mathrm{SH}$ is stronger than normal, more cold air from high latitudes and the polar region intrudes southward, resulting in a more frequent occurrence of WECDs in all of NC. The largescale atmospheric circulation anomalies are probably induced by more snow cover over northeastern Eurasia in the preceding autumn through the local positive feedback between the snow and SATs and the tropospheric-stratospheric interaction (Fig. 6), as suggested by some other studies (e.g., Cohen and Entekhabi 1999; Gong et al. 2002; Allen and Zender 2011). On the other hand, in the winter, when the negative AO cooccurs with the weaker $\mathrm{SH}$, eastern $\mathrm{NC}$ is still under the cold-air intrusion related to the negative $\mathrm{AO}$ and likely to have more WECDs, whereas western NC is impacted by the anomalous warm-air advection along the southeastern edge of the negative SLP anomalies related to the weaker SH and tends to have fewer WECDs. This forms the east-west contrasted anomalies of WECDs in NC. The weakening of the SH may be caused by the tropical SST anomalies relevant to El Niño. The northeastward shift of the Aleutian low introduced by the PNA teleconnection in the El Niño winters can displace the EAT northward. Therefore, the descending motion upstream of the climatological EAT and east of the Caspian Sea becomes weaker and results in a weaker $\mathrm{SH}$ (Fig. 9), which is consistent with the results of Leung and Zhou (2015, 2016) and Leung et al. (2017).

The reproducibility of the observed spatial patterns and possible causalities of the WECD variations in NC by the 10 coupled models participating in CMIP5 was assessed. All the models were able to reproduce the observed EOF1 and EOF2 modes (Fig. 10). The large-scale circulation anomalies related to EOF1/EOF2 were well simulated in most models; the multimodel ensemble of the SLP anomalies north of $15^{\circ} \mathrm{N}$ related to EOF1/EOF2 had a spatial correlation coefficient with respect to the observations higher than 0.75 , significant at the $99 \%$ confidence level using the two-tailed $t$ test (Fig. 12). In addition, most models reproduced well the possible impacts of the preceding autumn snow over northeastern Eurasia on EOF1 (Fig. 13a). The impacts of the ENSO on EOF2 were also simulated to some extent (Fig. 13b).

Thus far, we have mainly focused on the large-scale circulation anomalies in the lower troposphere related to the variations in the number of WECDs in NC because the colder-than-normal air in $\mathrm{NC}$ in winter is rarely locally formed but rather advected from higher latitudes by the lower-tropospheric circulation anomalies (e.g., Tao 1957; Ding 1990). This is also the case for some other places in the Eurasian continent (e.g., Thompson and Wallace 1998; Pfahl and Wernli 2012). However, the winter circulation anomalies in the lower troposphere are often closely coupled to those in the middle and upper levels. Indeed, when NC has WECD anomalies of the EOF1 pattern, the EAT is deepened and the subtropical jet is strengthened (Figs. 14a,b), analogous to the anomalous circulation configuration of a stronger EAWM (e.g., Yang et al. 2002; Kuang et al. 2008). In addition, there are largescale planetary waves propagating from the North Atlantic to East Asia, reminiscent of the Eurasian or Scandinavian patterns (Fig. 14a). Compared to the conventional three action centers of the Eurasian pattern, as denoted by the green dots in Fig. 14a, the anomalous centers of the planetary waves shift westward. Hence, the linear correlation coefficient between EOF1 and the winter Eurasian pattern indices calculated following Wallace and Gutzler (1981) is close to zero. Instead, EOF1 has a significant positive correlation coefficient of 0.42 at the $99 \%$ confidence level with the winter Scandinavian pattern index, which can be downloaded from the Climate Prediction Center of NOAA (https://www.cpc.ncep.noaa.gov/data/teledoc/scand.shtml). 
(a) Z500 regressed on EOF1

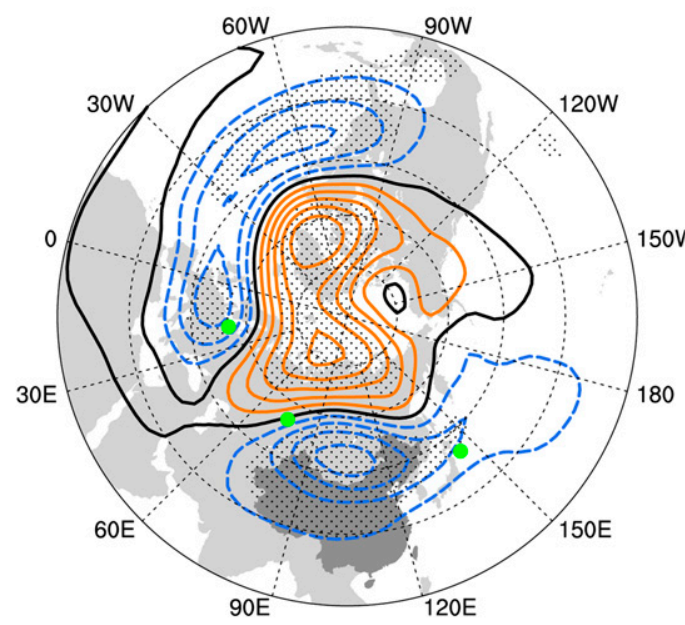

(c) Z500 regressed on snow

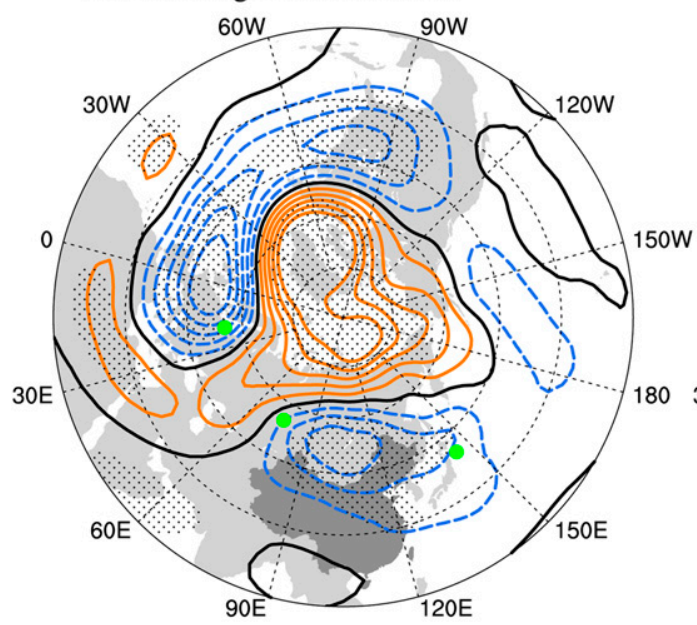

(b) U200 regressed on EOF1

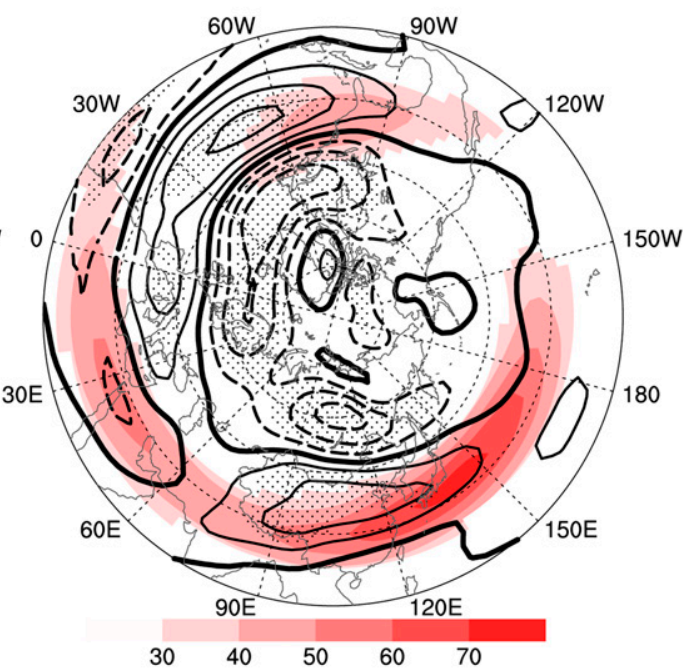

(d) U200 regressed on snow

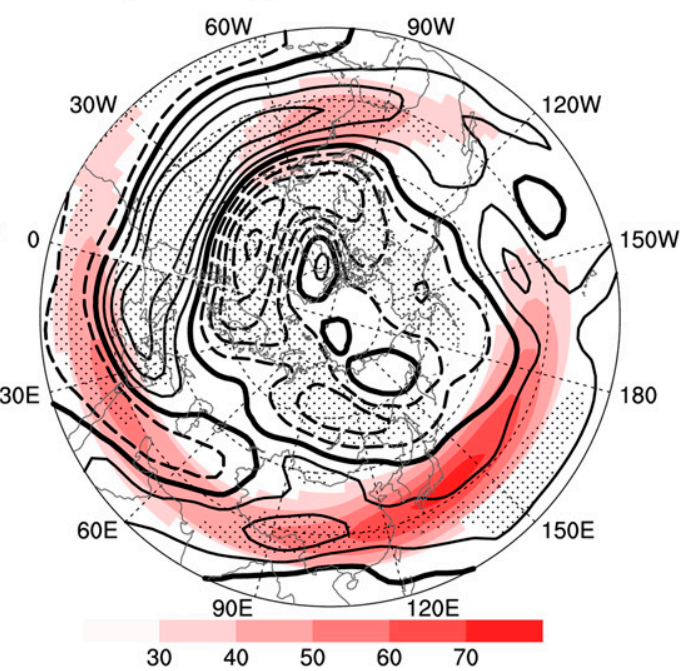

FIG. 14. December-February anomalies in (a),(c) the geopotential height at $500 \mathrm{hPa}$ (contours; interval: $5 \mathrm{gpm}$ ) and (b),(d) zonal wind at $200 \mathrm{hPa}$ (contours; interval: $2 \mathrm{~m} \mathrm{~s}^{-1}$ ) regressed on the time sequences of the observed (a),(b) EOF1 and (c),(d) September-November snow index. The anomalies significant at the 95\% confidence level are stippled. The green dots in (a) and (c) denote the conventional action centers of the Eurasian pattern. The red shading in (b) and (d) denotes the climatological 200-hPa zonal wind greater than $30 \mathrm{~m} \mathrm{~s}^{-1}$.

However, the statistically significant relation between EOF1 and the Scandinavian pattern may be attributed to the AO; the partial correlation coefficient between them drops to 0.18 after removing the covariance with the AO, while that between EOF1 and the AO remains -0.39 after removing the covariance with the Scandinavian pattern, still significant at the $99 \%$ confidence level. Nevertheless, the three-dimensional circulation anomalies related to EOF1 are closely related to the preceding autumn snow in northeastern Eurasia (Figs. 3a, 5b, 14), suggesting the substantial impacts of autumn snow on the subsequent WECDs in NC. Note that when NC has WECD anomalies of the EOF2 pattern, there are no prominent planetary waves propagating from the North Atlantic or western Europe to East Asia, and the strength of the subtropical jet in the upper level does not change significantly (figures not shown). This is probably because EOF2 is related to the co-occurrence of the weaker $\mathrm{SH}$ and the negative $\mathrm{AO}$ and because their circulation anomalies have competing influences on the EAWM. Hence, the large-scale circulation relevant to the EAWM in the 
middle and upper levels does not vary significantly. This indicates the dominant impacts of the lower tropospheric circulation on the formation of WECDs in NC.

In this study, the WECDs were selected as days during which the maximum temperatures are below or equal to the 10th percentile of all the winter daily maximum temperatures within the base period of 1961-90. However, nearly the same results can be attained if the daily minimum or averaged temperatures are adopted to define the WECDs. Hence, the SH and the AO are closely related to the spatiotemporal variations in the number of WECDs in NC. As discussed in the introduction section, it is controversial whether the circulation anomalies related to the AO impact the East Asian winter climate by modifying the strength of the SH (e.g., Gong et al. 2001; $\mathrm{Wu}$ and Wang 2002). From the results of the current study, no matter when the negative $\mathrm{AO}$ co-occurs with the stronger or weaker $\mathrm{SH}$, eastern $\mathrm{NC}$ always has more WECDs (Figs. 4f, 7a), indicating that the AO-related circulation anomalies can impact at least part of East Asia independently of the SH.

This study reveals the large-scale circulation anomalies and possible causalities related to the variations in the number of WECDs in NC by using the observed and reanalysis data. Their reproducibility is also assessed in the 10 CMIP5 models. The results shown here not only improve our knowledge of the extreme events in $\mathrm{NC}$ but also suggest that the use of these models, especially the multimodel ensemble, to simulate and predict WECDs in $\mathrm{NC}$ may be feasible. In addition to extreme cold events, $\mathrm{NC}$ also suffers from drought and heatwaves. More studies are needed to investigate their spatiotemporal variations and large-scale drivers to enhance our understanding of weather and climate extremes in China.

Acknowledgments. The authors are very thankful to three anonymous reviewers for their constructive comments that help improve the research substantially. All figures were drawn by NCL. The research is supported by the National Key R\&D Program of China 2016YFA0600702 and 2016YFA0600402, National Natural Science Foundation of China 41875099, and the funding of Jiangsu innovation and entrepreneurship team.

\section{REFERENCES}

Allen, R. J., and C. S. Zender, 2011: Forcing of the Arctic Oscillation by Eurasian snow cover. J. Climate, 24, 6528-6539, https://doi.org/10.1175/2011JCLI4157.1.

Barnston, A. G., and R. E. Livezey, 1987: Classification, seasonality and persistence of low-frequency atmospheric circulation patterns. Mon. Wea. Rev., 115, 1083-1126, https://doi.org/ 10.1175/1520-0493(1987)115<1083:CSAPOL > 2.0.CO;2.
Bjerknes, J., 1969: Atmospheric teleconnections from the equatorial Pacific. Mon. Wea. Rev., 97, 163-172, https://doi.org/ 10.1175/1520-0493(1969)097<0163:ATFTEP >2.3.CO;2.

Boyle, J. S., and T. J. Chen, 1987: Synoptic aspects of the wintertime East Asian monsoon. Monsoon Meteorology, C. P. Chang and T. N. Krishnamurti, Eds., Oxford University Press, 126160.

Bueh, C., and H. Nakamura, 2007: Scandinavian pattern and its climatic impact. Quart. J. Roy. Meteor. Soc., 133, 2117-2131, https://doi.org/10.1002/qj.173.

Chen, S., B. Yu, and W. Chen, 2014: An analysis on the physical process of the influence of AO on ENSO. Climate Dyn., 42, 973-989, https://doi.org/10.1007/s00382-012-1654-z.

Chen, W., and L. Kang, 2006: Linkage between the Arctic Oscillation and winter climate over East Asia on the interannual timescale: Roles of quasi-stationary planetary waves (in Chinese). Chin. J. Atmos. Sci., 30, 863-870.

Cheung, H. N., W. Zhou, H. Y. Mok, and M. C. Wu, 2012: Relationship between Ural-Siberian blocking and the East Asian winter monsoon in relation to the Arctic Oscillation and the El Niño-Southern Oscillation. J. Climate, 25, 4242-4257, https:// doi.org/10.1175/JCLI-D-11-00225.1.

,,,$----\frac{1}{-}$, and Y. Shao, 2013: Revisiting the climate of atmospheric blocking in the Northern Hemisphere. $A d v$. Atmos. Sci., 30, 397-410, https://doi.org/10.1007/s00376-0122006-y.

- — , Y. T. Leung, C. M. Shun, S. M. Lee, and H. W. Tong, 2016: A strong phase reversal of the Arctic Oscillation in midwinter 2015/2016: Role of the stratospheric polar vortex and tropospheric blocking. J. Geophys. Res. Atmos., 121, 13 443-13 457, https://doi.org/10.1002/2016JD025288.

Cohen, J., and D. Entekhabi, 1999: Eurasian snow cover variability and Northern Hemisphere climate predictability. Geophys. Res. Lett., 26, 345-348, https://doi.org/10.1029/1998GL900321.

-, M. Barlow, P. J. Kushner, and K. Saito, 2007: Stratospheretroposphere coupling and links with Eurasian land surface variability. J. Climate, 20, 5335-5343, https://doi.org/10.1175/ 2007JCLI1725.1.

—, J. C. Furtado, J. Jones, M. Barlow, D. Whittleston, and D. Entekhabi, 2014: Linking Siberian snow cover to precursors of stratospheric variability. J. Climate, 27, 5422-5431, https:// doi.org/10.1175/JCLI-D-13-00779.1.

Dai, X.-G., C.-B. Fu, and P. Wang, 2005: Interdecadal change of atmospheric stationary waves and North China drought. Chin. Phys., 14, 850-858, https://doi.org/10.1088/1009-1963/14/4/038.

Ding, Y., 1990: A statistical study of winter monsoon in East Asia (in Chinese). J. Trop. Meteor., 6, 119-128.

, and T. N. Krishnamurti, 1987: Heat budget of the Siberian high and the winter monsoon. Mon. Wea. Rev., 115, 24282449, https://doi.org/10.1175/1520-0493(1987)115<2428: HBOTSH $>2.0 . \mathrm{CO} ; 2$.

- S. Wen, and Y. Li, 1994: A study of dynamic structures of Siberian highs in winter (in Chinese). Acta Meteor. Sin., 52, 430-439.

Easterling, D. R., J. L. Evans, P. Ya. Groisman, T. R. Karl, K. E. Kunkel, and P. Ambenje, 2000: Observed variability and trends in extreme climate events: A brief review. Bull. Amer. Meteor. Soc., 81, 417-425, https://doi.org/10.1175/1520-0477(2000) 081<0417:OVATIE $>2.3$.CO;2.

Estilow, T. W., A. H. Young, and D. A. Robinson, 2015: A longterm Northern Hemisphere snow cover extent data record for climate studies and monitoring. Earth Syst. Sci. Data, 7, 137142, https://doi.org/10.5194/essd-7-137-2015. 
Fan, Y., and H. van den Dool, 2008: A global monthly land surface temperature analysis for 1948-present. J. Geophys. Res., 113, D01103, https://doi.org/10.1029/2007JD008470.

Garfinkel, G. I., and D. L. Hartmann, 2008: Different ENSO teleconnections and their effects on the stratospheric polar vortex. J. Geophys. Res., 113, D18114, https://doi.org/10.1029/ 2008JD009920.

Gong, D., S. Wang, and J. Zhu, 2001: East Asian winter monsoon and Arctic Oscillation. Geophys. Res. Lett., 28, 2073-2076, https://doi.org/10.1029/2000GL012311.

Gong, G., D. Entekhabi, and J. Cohen, 2002: A large-ensemble model study of the wintertime AO-NAO and the role of interannual snow perturbations. J. Climate, 15, 3488-3499, https://doi.org/ 10.1175/1520-0442(2002)015<3488:ALEMSO>2.0.CO;2.

Guo, Q., 1994: Relationship between the variations of East Asian winter monsoon and temperature anomalies in China (in Chinese). J. Appl. Meteor. Sci., 5, 218-225.

IPCC, 2013: Climate Change 2013: The Physical Science Basis. T. F. Stocker et al., Eds., Cambridge University Press, 1535 pp.

Jeong, J., and C. Ho, 2005: Changes in occurrence of cold surges over East Asia in association with Arctic Oscillation. Geophys. Res. Lett., 32, L14704, https://doi.org/10.1029/2005GL023024.

Jhun, J., and E. Lee, 2004: A new East Asian winter monsoon index and associated characteristics of the winter monsoon. J. Climate, 17, 711-726, https://doi.org/10.1175/1520-0442(2004)017<0711: ANEAWM $>2.0 . \mathrm{CO} ; 2$.

Kalnay, and Coauthors, 1996: The NCEP/NCAR 40-Year Reanalysis Project. Bull. Amer. Meteor. Soc., 77, 437-471, https:// doi.org/10.1175/1520-0477(1996)077<0437:TNYRP > 2.0.CO;2.

Kuang, X., Y. Zhang, and J. Liu, 2008: Relationship between subtropical upper-tropospheric westerly jet and East Asian winter monsoon (in Chinese). Plateau Meteor., 27, 701-712.

Kumar, A., and F. Yang, 2003: Comparative influence of snow and SST variability on extratropical climate in northern winter. J. Climate, 16, 2248-2261, https://doi.org/10.1175/2771.1.

Leung, Y. T., and W. Zhou, 2015: Variation of circulation and East Asian climate associated with anomalous strength and displacement of the East Asian trough. Climate Dyn., 45, 2713 2732, https://doi.org/10.1007/s00382-015-2504-6.

_ and - 2016: Direct and indirect ENSO modulation of winter temperature over the Asian-Pacific-American region. Sci. Rep., 6, 36356, https://doi.org/10.1038/SREP36356.

- H. Heung, and W. Zhou, 2017: Meridional displacement of the East Asian trough and its response to the ENSO forcing. Climate Dyn., 48, 335-352, https://doi.org/10.1007/s00382-0163077-8.

Li, F., H. Wang, and Y. Gao, 2014: On the strengthened relationship between the East Asian winter monsoon and Arctic Oscillation: A comparison of 1950-70 and 1983-2012. J. Climate, 27, 5075-5091, https://doi.org/10.1175/JCLI-D-13-00335.1.

Li, Y., and N.-C. Lau, 2013: Influences of ENSO on stratospheric variability, and the descent of stratospheric perturbations into the lower troposphere. J. Climate, 26, 4725-4748, https:// doi.org/10.1175/JCLI-D-12-00581.1.

Ma, T., Z. Wu, and Z. Jiang, 2012: How does coldwave frequency in China respond to a warming climate? Climate Dyn., 39, $2487-$ 2496, https://doi.org/10.1007/s00382-012-1354-8.

McPhaden, M. J., S. E. Zebiak, and M. H. Glantz, 2006: ENSO as an integrating concept in Earth science. Science, 314, 17401745, https://doi.org/10.1126/science.1132588.

Nakamura, T., T. Tachibana, M. Honda, and S. Yamane, 2006: Influence of the Northern Hemisphere annular mode on
ENSO by modulating westerly wind bursts. Geophys. Res. Lett., 33, L07709, https://doi.org/10.1029/2005GL025432.

North, G. R., T. L. Bell, R. F. Cahalan, and F. J. Moeng, 1982: Sampling errors in the estimation of empirical orthogonal functions. Mon. Wea. Rev., 110, 699-706, https://doi.org/ 10.1175/1520-0493(1982)110<0699:SEITEO > 2.0.CO;2.

Overland, J. E., J. M. Adams, and N. A. Bond, 1999: Decadal variability of the Aleutian low and its relation to high-latitude circulation. J. Climate, 12, 1542-1548, https://doi.org/10.1175/ 1520-0442(1999)012<1542:DVOTAL > 2.0.CO;2.

Pfahl, S., and H. Wernli, 2012: Quantifying the relevance of atmospheric blocking for co-located temperature extremes in the Northern Hemisphere on (sub-) daily time scales. Geophys. Res. Lett., 39, L12807, https://doi.org/10.1029/2012GL052261.

Ratnam, J. V., and Coauthors, 2016: Anatomy of Indian heatwaves. Sci. Rep., 6, 24395, https://doi.org/10.1038/srep24395.

Rayner, N. A., and Coauthors, 2003: Global analyses of sea surface temperature, sea ice, and night marine air temperature since the late nineteenth century. J. Geophys. Res., 108, 4407, https://doi.org/10.1029/2002JD002670.

Seager, R., Y. Kushnir, C. Herweijer, N. Naik, and J. Velez, 2005: Modeling of tropical forcing of persistent droughts and pluvials over western North America: 1856-2000. J. Climate, 18, 4065-4088, https://doi.org/10.1175/JCLI3522.1.

Sillmann, J., and Coauthors, 2017: Understanding, modeling and predicting weather and climate extremes: Challenges and opportunities. Wea. Climate Extremes, 18, 65-74, https:// doi.org/10.1016/j.wace.2017.10.003.

Song, C. L., and Coauthors, 2010: Yearbook of Chinese Meteorological Disasters in China (in Chinese). China Meteorological Press, $105-167$.

Song, L., and R. Wu, 2017: Processes for occurrence of strong cold events over eastern China. J. Climate, 30, 9247-9266, https:// doi.org/10.1175/JCLI-D-16-0857.1.

Sung, M.-K., G.-H. Lim, W.-T. Kwon, K.-O. Boo, and J.-S. Kug, 2009: Short-term variation of Eurasian pattern and its relation to winter weather over East Asia. Int. J. Climatol., 29, 771-775, https://doi.org/10.1002/joc.1774.

Suo, L., J. Huang, and B. Tang, 2008: The influence of winter Arctic Oscillation on maximum and minimum air temperature over China in winter (in Chinese). J. Trop. Meteor., 24, 163-168.

Takaya, K., and H. Nakamura, 2001: A formulation of a phaseindependent wave-activity flux for stationary and migratory quasigeostrophic eddies on a zonally varying basic flow. $J$. Atmos. Sci., 58, 608-627, https://doi.org/10.1175/15200469(2001)058<0608:AFOAPI > 2.0.CO;2.

$\longrightarrow$, and — 2013: Interannual variability of the East Asian winter monsoon and related modulations of the planetary waves. J. Climate, 26, 9445-9461, https://doi.org/10.1175/JCLID-12-00842.1.

Tao, S., 1957: A synoptic and aerological study on a cold wave in the Far East during the period of the breakdown of the blocking situation over Eurasian and Atlantic (in Chinese). Acta Meteor. Sin., 28, 63-74.

_ , and Q. Zhang, 1998: Response of the Asian winter and summer monsoon to ENSO events (in Chinese). Chin. J. Atmos. Sci., 22, 399-407.

Taylor, K. E., R. J. Stouffer, and G. A. Meehl, 2012: An overview of CMIP5 and the experiment design. Bull. Amer. Meteor. Soc., 93, 485-498, https://doi.org/10.1175/BAMS-D-11-00094.1.

Thompson, D. W. J., and J. M. T. Wallace, 1998: The Arctic Oscillation signature in the wintertime geopotential height and 
temperature fields. Geophys. Res. Lett., 25, 1297-1300, https:// doi.org/10.1029/98GL00950.

— , and — , 2000: Annular modes in the extratropical circulation, Part I: Month-to-month variability. J. Climate, 13, 1000-1016, https://doi.org/10.1175/1520-0442(2000) 013<1000:AMITEC > 2.0.CO;2.

Wallace, J. M., and D. S. Gutzler, 1981: Teleconnections in the geopotential height field during the Northern Hemisphere winter. Mon. Wea. Rev., 109, 784-812, https://doi.org/10.1175/ 1520-0493(1981)109<0784:TITGHF>2.0.CO;2.

Walland, D. J., and I. Simmonds, 1996: Modeled atmospheric response to changes in Northern Hemisphere snow cover. $\mathrm{Cli}$ mate Dyn., 13, 25-34, https://doi.org/10.1007/s003820050150.

Walsh, J. E., and B. Ross, 1988: Sensitivity of 30-day dynamical forecasts to snow cover. J. Climate, 1, 739-754, https://doi.org/ 10.1175/1520-0442(1988)001<0739:SODDFT $>2.0 . C O ; 2$

Wang, B., R. Wu, and X. Fu, 2000: Pacific-East Asian teleconnection: How does ENSO affect East Asian climate? J. Climate, 13, 1517-1536, https://doi.org/10.1175/1520-0442(2000)013<1517: PEATHD $>2.0$.CO;2.

Wang, L., W. Chen, W. Zhou, and R. Huang, 2009: Interannual variations of East Asian trough axis at $500 \mathrm{hPa}$ and its association with the East Asian winter monsoon pathway. J. Climate, 22, 600-614, https://doi.org/10.1175/2008JCLI2295.1.

Wang, N., and Y. Zhang, 2015: Evolution of Eurasian teleconnection pattern and its relationship to climate anomalies in China. Climate Dyn., 44, 1017-1028, https://doi.org/10.1007/ s00382-014-2171-z.

Watanabe, M., and T. Nitta, 1999: Decadal changes in the atmospheric circulation and associated surface climate variations in the Northern Hemispheric winter. J. Climate, 12, 494-510, https:// doi.org/10.1175/1520-0442(1999)012<0494:DCITAC > 2.0.CO;2.

Wei, K., W. Chen, and W. Zhou, 2011: Changes in the East Asian cold season since 2000. Adv. Atmos. Sci., 28, 69-79, https:// doi.org/10.1007/s00376-010-9232-y.
Wen, M., S. Yang, A. Kumar, and P. Zhang, 2009: An analysis of the large-scale climate anomalies associated with the snowstorms affecting China in January 2008. Mon. Wea. Rev., 137, 11111131, https://doi.org/10.1175/2008MWR2638.1.

Wu, B., and J. Wang, 2002: Winter Arctic Oscillation, Siberian high and East Asian winter monsoon. Geophys. Res. Lett., 29, 1897, https://doi.org/10.1029/2002GL015373.

_ K. K. Yang, and J. A. Francis, 2017: A cold event in Asia during January-February 2012 and its possible association with Arctic Sea ice loss. J. Climate, 30, 7971-7990, https://doi.org/ 10.1175/JCLI-D-16-0115.1.

Yang, S., K. M. Lau, and K. M. Kim, 2002: Variations of the East Asian jet stream and Asian-Pacific-American winter climate anomalies. J. Climate, 15, 306-325, https://doi.org/10.1175/ 1520-0442(2002)015<0306:VOTEAJ > 2.0.CO;2.

You, Q., and Coauthors, 2011: Changes in daily climate extremes in China and their connection to the large scale atmospheric circulation during 1961-2003. Climate Dyn., 36, 2399-2417, https://doi.org/10.1007/s00382-009-0735-0.

Zhang, J., 2001: January 2001 low temperature in the northeastern China and heavy snowstorm over Inner Mongolia and Xinjiang (in Chinese). Mon. Meteor., 27, 62-63.

Zhang, R., A. Sumi, and M. Kimoto, 1996: Impact of El Niño on the East Asian monsoon: A diagnostic study of the '86/87 and '91/92 events. J. Meteor. Soc. Japan, 74, 49-62, https://doi.org/ 10.2151/jmsj1965.74.1_49.

Zhang, Y., K. R. Sperber, and K. R. Boyle, 1997: Climatology and interannual variation of the East Asian winter monsoon: Results from the 1979-95 NCEP/NCAR reanalysis. Mon. Wea. Rev., 125, 2605-2619, https://doi.org/10.1175/1520-0493(1997) 125<2605:CAIVOT $>2.0$. CO;2.

Zhou, W., J. C. L. Chan, W. Chen, J. Ling, J. G. Pinto, and Y. P. Shao, 2009: Synoptic-scale controls of persistent low temperature and icy weather over southern China in January 2018. Mon. Wea. Rev., 137, 3978-3911, https://doi.org/10.1175/ 2009MWR2952.1. 